\title{
Contracting Out Public Service Provision to Not-for-Profit Firms
}

\author{
April 2007
}

\begin{abstract}
In an incomplete contract setting, we analyze the contracting out of public service provision, comparing the performance of for-profit and not-forprofit private firms. Two institutional arrangements are considered, control rights lying either with the firm as under the UK's Private Finance Initiative (PFI) or the government (as under traditional procurement).
\end{abstract}

JEL Classification: H41, L31, L33.

Keywords: contracting out, not-for-profit firms, private finance initiative, public-private partnership, incomplete contracts, public service provision. 


\section{Introduction}

Recent years have witnessed a steady stream of innovations in the way public services are provided. In the UK, under the Private Finance Initiative (PFI), it has become common for the government to contract out the provision of public services to a consortium of private firms that designs, finances, builds and manages the facilities concerned (HM Treasury, 2003). In Canada, such public-private partnerships have been used for major infrastructure projects, such as the 407 Express Toll Route to the north of Toronto and the redevelopment of Pearson International Airport (Daniels and Trebilcock, 2000), while in the US, in much of the European Union, and in developing economies, there has been increasing use of similar schemes (Linder and Rosenau, 2000). This approach contrasts sharply with the way public services have traditionally been procured. Under traditional procurement, the government specifies the inputs and retains control rights over how the service is delivered. Instead, under PFI, the government specifies the

output, that is, it specifies a basic service standard, but it is the firm that has control rights over how to deliver the service.

Not-for-profit firms (NPs) have long been established in public service provision, for example in health and education. However, there has recently been an extensively-debated expansion in the role of NPs (see Bennett et al., 2003; IPPR 2003, and Weisbrod 1997). An important recent example in the UK is the responsibility for rail track facilities that the government has given to the NP, Network Rail. Among the other well-publicized cases are Glas Cymru, which was created 
on a private initiative in April 2000 as a holding company for the assets of Dwr Cymru, the Welsh water utility, and NAV Canada, which was established in 1996, and owns and operates Canada's civil air navigation service.

In this paper, we analyze the contracting out of service provision to private firms, and we compare the case in which the contractor is an NP to that in which it is a for-profit firm (FP). We consider these cases under two different institutional arrangements. The first is traditional procurement, under which the government retains control rights over the project; the second is PFI, the firm having control rights. We take an incomplete-contract approach (see, e.g., Hart, 1995), building on the seminal work on public service provision by Hart, Shleifer and Vishny (1997). We assume that the firm may make an observable but unverifiable investment, researching innovative approaches to perform its task in excess of the basic standard specified in the initial contract. We assume that an innovation, if implemented, has an effect both on the social benefit that is generated by the production of the public service and on the firm's profit. Control rights (i.e., ownership of the project) give the power of veto over the implementation of any given innovation.

Whereas an FP may be assumed to maximize profits, an NP operates under a non-distribution constraint, which bans it from redistributing profit to its members. Also, an may be founded with a specific mission in mind, and users and stakeholders participate on its board of trustees. To capture these considerations as simply as possible, we assume that the NP's objective is to maximize benefits, though subject to a profit constraint. Such a constraint is particularly important 
for an NP because, unlike an FP, it does not have the option of raising funds on the stock market. This approach contrasts with that of Glaeser and Shleifer (2001), who include profits directly in the NP's objective function, but with a lower value attached than is the case for an FP: profits are valuable to the NP only insofar as they permit consumption of perquisites and the building up of precautionary financial reserves.

We compare the investment incentives of an FP and an NP under different institutional arrangements, noting the implications for different types of public services. We consider three alternative scenarios. In the first, implementation of an investment increases both the contractor's profit and social benefit (we refer to this as 'profitable quality improvement'). For example, the investment may be in asset quality (e.g., of a hospital or a school building) that generates both lower maintenance costs for the contractor and greater social benefit from the use of the asset for public service provision (e.g., fewer disruptions to teaching or a better healing environment). In contrast, the second and the third scenarios are characterized by a conflict between social benefit and profit. In the second ('unprofitable quality improvement') this occurs because implementation of an investment that increases social benefit is costly and, in the absence of a side-payment from the government, will cut the contractor's profit. For example, implementation of the investment might improve safety, but the original contract may not offer scope to raise revenue to cover the costs of implementation. In the third ('cost cutting at the expense of quality') implementation of an investment increases profit, but has an adverse impact on social benefit. For example, a cost-cutting innovation might 
compromise safety. We show that

Our results show that the appropriate institutional arrangement depends on which scenario obtains, can be summarized as follows...

The theoretical literature on the provision of public services is expanding rapidly. Hart, Shleifer and Vishny (1997), Schmitz (2000), and King and Pitchford (2001) compare public provision with contracting out to an FP. The optimality of bundling building and managing operations in PFI projects with FPs is discussed by Bennett and Iossa (2006) and Hart (2002) under incomplete contracts, and by Bentz, Grout and Halonen (2001) under complete contracts. Bundling in an incomplete-contract model is also analyzed by Bös and De Fraja (2002), who examine the case of health care for which quality is unverifiable. However, none of these papers considers public service provision by NPs.

There is also an extensive literature on NPs, though, for many years, its main focus was on the relationship between the firm and its donors (see e.g. RoseAckerman, 1996). However, a related branch of the literature considers NPs that do not rely on donations (see Hansmann, 1986, 1996). A recent formulation by Glaeser and Shleifer (2001) examines why an entrepreneur setting up a firm might prefer to make it an NP. In their model, as in ours, an NP generates perquisites for an entrepreneur that are not as valuable as income, so that, relative to a $\mathrm{FP}$, the NP has weaker profit incentives. Closer to our work is that of Besley and Ghatak (2001). In their model, as in ours, a critical role is played by the service provider's valuation of social benefit. They show that control rights should be left with the party that values services more highly, thus indicating a role for 'benevolent' NPs. 
However, contrary to us, they do not consider PFI - which is shown in our analysis to widen the potential role for NPs in effective public service provision.

The paper is organized as follows. Section 2 outlines the model, while in Section 3 we compare the effects of the different institutional arrangements. Section 4 provides some extensions and Section 5 concludes.

\section{The Model}

We consider a setting where, initially, the government and the firm agree a contract that specifies observable and verifiable basic standards for the provision of a public service. However, before operations begin, the firm may make an observable but unverifiable investment, which we denote by $x \geq 0$, researching innovative approaches to performing its task in excess of the basic standard. The cost of this investment in monetary terms is $C(x)$, which, for simplicity, we shall assume that it is quadratic: $C(x)=x^{2} / 2$. The investment cannot be contracted upon ex ante, for it is not possible to specify in advance the delivery of a specific innovation. We assume that an innovation, if implemented, affects both the profit and the social benefit generated by the provision of the public service. In our solutions, innovation $x$ is implemented, and so we economize on notation by writing social benefit and profit as functions of $x$.

The social benefit generated by the provision of the public service is

$$
B(x)=B_{0}+\beta b x ; \quad(b>0)
$$

where $B_{0}$ is a positive constant denoting verifiable basic standards and $\beta$ is a shift 
parameter whose value is either 1 or -1 . If $\beta=1, x$ increases social benefit; if $\beta=-1, x$ decreases social benefit.

Gross profit is defined to be

$$
\Pi(x)=\Pi_{0}+\gamma \pi x ; \quad(\pi>0)
$$

where $\Pi_{0}$ is the default profit that the firm would obtain if it set $x=0 . \gamma$ is a shift parameter whose value is either 1 or -1 : if $\gamma=1, x$ increases profit;. if $\gamma=-1, x$ decreases profit. We assume that $B(x)$ and $\Pi(x)$ are observable but unverifiable.

Net profit $\hat{\Pi}(x)$ is defined also to include the investment cost $C(x)$ and the monetary transfer $z$ that is received from the government should bargaining occur in order to get the firm to institute the innovation $x$. Thus,

$$
\hat{\Pi}(x)=\Pi_{0}+\gamma \pi x-C(x)+z .
$$

An FP chooses $x$ to maximize net profit $\hat{\Pi}(x)$. An NP chooses $x$ so as to maximize benefits, subject to a net profit constraint; that is, its objective function is

$$
\max _{x} B(x) \text { subject to } \Pi_{0}+\gamma \pi x-C(x)+z \geq \bar{\Pi} .
$$

$\bar{\Pi}$ is a parameter which, for now, we assume equals $\Pi_{0}$, implying that, if we disregard the profit $\Pi_{0}$ that would be achieved by satisfying basic standards, any further profit $\gamma \pi x-C(x)+z$ (the profit related to innovation) must be non-negative.

The government is assumed to maximize $B(x)-z_{0}-z$, where $z_{0}$ is the payment it makes for satisfying basic standards; that is, it maximizes benefits minus any payments to the firm. ${ }^{1}$

\footnotetext{
${ }^{1}$ We are assuming here that 'the government' is a government agency, such as a local govern-
} 
We focus on the following three scenarios (examples of which are discussed in Section 4):

(a) Profitable quality improvement: implementation of innovation $x$ raises both social benefit and the firm's profit $(\beta=\gamma=1)$.

(b) Unprofitable quality improvement: implementation of innovation $x$ raises social benefit but cuts the firm's profit $(\beta=1, \gamma=-1)$.

(c) Cost cutting at the expense of quality: implementation of innovation $x$ raises the firm's profit but cuts social benefit $(\beta=-1, \gamma=1)$.

We compare two institutional arrangements: the private finance initiative (PFI) and traditional procurement. We assume that under PFI the firm has control rights over the project, being free to implement the innovation without consulting the government. Under traditional procurement, however, the government has control rights over the project, and if there are gains from implementing the innovation, bargaining between the firm and the government will take place. We assume the simplest possible form of alternating offers bargaining is used, with each side having a chance that it will be the one to make the offer. The other side then chooses whether to accept the offer, with implementation of the innovation taking place, or it rejects the offer, in which case there is no implementation. Thus, we assume that the government makes the offer with probability $\lambda \in[0,1]$, while the firm makes the offer with probability $1-\lambda .^{2}$ In the absence of a budget constraint the solution is then equivalent to a Nash bargain with bargaining weights of $\lambda$ and ment or ministry, with its own objectives, rather than an abstract welfare-maximizing government.

${ }^{2}$ Reference justifying this approach. 
$1-\lambda$. For simplicity, we assume for now that $\lambda=1 / 2$.

As a benchmark, we specify the first-best solution. We define welfare $W(x)$ to be the sum of benefits, gross profits and (negatively) the investment cost; that is,

$$
W(x)=B(x)+\Pi(x)-C(x) .
$$

The first-best investment $x^{*}$ maximizes $W(x)$. We assume for now that $b>\pi$, that is, the dominant effect of an innovation is on benefits. (The reverse of this inequality is considered in the next section.) $x^{*}$ is therefore given, for the respective cases, by

$$
\begin{aligned}
& \text { (a) } x^{*}=b+\pi ; \\
& \text { (b) } x^{*}=b-\pi ; \\
& \text { (c) } x^{*}=0 .
\end{aligned}
$$

In this setting, for each institutional arrangement, PFI and traditional procurement, and for each type of firm, FP or NP, we compare investment levels, and thus welfare levels. The timing of the game is as follows. In period 0 the government sets the basic standards of service provision. In period 1 the contractor (FP or NP) undertakes investment $x$ researching improved methods for performing its task in excess of the basic standards. In period 2, if the government has control rights (traditional procurement), and if there would be gains from implementing the innovation, bargaining between the firm and the government takes place; if instead the firm has control rights (PFI), it is free to implement the innovation without consulting the government. In period 3 the service is provided. 


\subsection{PFI}

We assume in this section that the firm has control rights over the project. We consider what happens to investment first when the firm is FP and then when it is NP.

When the firm is an FP it chooses $x$ to maximize $\hat{\Pi}(x)$, as given by (2). Thus, writing $x_{p}^{F}$ for the level of $x$ it chooses, we have

$$
\begin{aligned}
& \text { (a) } x_{p}^{F}=\pi ; \\
& \text { (b) } x_{p}^{F}=0 ; \\
& \text { (c) } x_{p}^{F}=\pi
\end{aligned}
$$

In cases (a) and (c), with profit is increasing in $x$, an interior solution obtains; and in case (b), with profits decreasing in $x$, the FP does not invest. In each case the solution is different to the first-best because the FP does not take into account the effect of $x$ on benefits. In cases (a) and (b) $x_{p}^{F}<x^{*}$ since the FP does not take into account the positive effect of $x$ on $B$; in case (c) $x_{p}^{F}>x^{*}$ since the FP does not take into account the negative effect of $x$ on benefits.

Suppose now that the firm is an NP, maximizing $B(x)$ subject to its budget constraint. Denote its investment by $x=x_{p}^{N}$. In case (a) both benefits and gross profit are increasing in $x$, and so the NP invests up to the point at which the budget constraint is binding; that is, $\pi x_{p}^{N}-C\left(x_{p}^{N}\right)=0$. Thus,

$$
\text { (a) } x_{p}^{N}=2 \pi \text {. }
$$

In case (b), although $x$ increases benefits, it is unprofitable, while in case (c) $x$ 
decreases benefits. Therefore the NP does not invest in either case:

$$
(\mathrm{b}, \mathrm{c}) x_{p}^{N}=0
$$

Compared to the first-best, it is seen that in case (a) there is underinvestment, given that $b>\pi$. Since $B$ is increasing in $x$, the NP invests up to where the budget constraint binds. Since $\pi$ is low, compared to $b$, the budget constraint binds at a point from which welfare could have been raised by further increasing $x$, but the budget constraint prevents the NP from doing so. In case (b) the first-best involves positive investment since $b>\pi$; but the budget constraint prevents any investment, and so there is underinvestment compared to the first-best. In case (c) $x_{p}^{N}=x^{*}$ since both are zero.

These conclusions lead immediately to our first proposition.

Proposition 1 Consider PFI for $b>\pi$ and $\bar{\Pi}=\Pi_{0}$. In case (a) $x_{p}^{F}<x_{p}^{N}<x^{*}$; there is underinvestment under both arrangements, but investment and welfare is greater with an NP than with an FP. In case (b) $x_{p}^{F}=x_{p}^{N}<x^{*}$; there is the same amount of underinvestment under each arrangement. In case (c) $x_{p}^{F}>x_{p}^{N}=$ $x^{*}$; the provision by the NP yields the first-best level of investment, but there is overinvestment by an FP. Taking the three cases together, the NP weakly dominates the FP in welfare terms.

\subsection{Traditional Procurement}

We now turn to traditional procurement, the government having control rights. Then an innovation cannot be implemented without the government's approval. If 
there are positive gains from implementation, it is reasonable to expect bargaining between the firm and the government to occur. We assume that the outside option is zero for each player. Hence, if bargaining occurs the default payoff for each player is the payoff that would obtain if there were no implementation of $x$ and only the basic standards were achieved. Thus, respective the default payoffs are $B_{0}-z_{0} \equiv V_{0}$ for the government, $\pi_{0}$ for an FP and $B_{0}$ for an NP.

Suppose first that the firm is an FP. With bargaining, if the FP makes the offer, the best it can do is ask the government to pay the amount that makes the government indifferent between agreeing to the offer or not; that is, the offer the FP makes is $z=\beta b x$. If the government makes the offer, the best it can do is ask the FP to pay the amount for which the FP is indifferent between accepting or not; that is, the government makes the offer $z=-\gamma \pi x .^{3}$ Hence, given the simple formulation of alternating-offers bargaining, there is an equal chance that $\hat{\Pi}(x)=\Pi_{0}+\gamma \pi x-C(x)+\beta b x$ or $\hat{\Pi}(x)=\Pi_{0}+\gamma \pi x-C(x)-\gamma \pi x=\Pi_{0}-C(x)$. Thus, $E[\hat{\Pi}(x)]=\Pi_{0}+\frac{1}{2}(\gamma \pi x+\beta b x)-C(x)$. We therefore have that in cases (a) and (b) the FP will set $d E[\hat{\Pi}(x)] / d x=\frac{1}{2}[\gamma \pi+\beta b]-x=0$. In case (c) $E[\hat{\Pi}(x)]=\Pi_{0}+\frac{1}{2}(\pi-b) x-C(x)$, which, for $b>x$, is decreasing in $x$. We therefore have that

$$
\begin{aligned}
& \text { (a) } x_{t}^{F}=\frac{1}{2}(\pi+b) ; \\
& \text { (b) } x_{t}^{F}=\frac{1}{2}(b-\pi) ; \\
& \text { (c) } x_{t}^{F}=0 .
\end{aligned}
$$

\footnotetext{
${ }^{3}$ Recall that the cost $C(x)$ has already been incurred here, so the government does not have to take $C(x)$ into account in its offer.
} 
Compared to the first-best, there is underinvestment in cases (a) and (b). If the FP makes the offer, it asks the government to pay the value of benefits from implementation, which, if this offer were going to be accepted, would cause the FP to internalize benefits fully and therefore the first-best would be achieved. However, if the government makes the offer it asks the FP to pay the amount of profits that result from implementation. If this offer is accepted, the FP will not earn these profits, and therefore it will internalize neither the profits nor the value of benefits. It is because there is a $50 \%$ chance that the government will make the offer that the FP's investment is below the first-best level. However, case (c) coincides with the first-best solution.

Suppose, instead, that the firm is an NP. Then, as far as it can, it will exploit its budget constraint to extract money from the government that can be used to increase benefits. With $\beta=1$, that is, in cases (a) and (b), the maximum it can extract is found by setting the government's default payoff $V_{0}=B_{0}-z_{0}$ equal to $B_{0}+b x-z_{0}-z$; that is, $z=b x$. Thus, if it chooses $x$ such that the budget constraint binds at $z=b x$, the government will pay it this amount. It is not relevant here which player makes the offer, for there is only one value of $z$ that is acceptable to both players. Substituting $z=b x$ into the NP's budget constraint, we have $b x+\gamma \pi x-x^{2} / 2=0$. Given also that in case (c) the NP will choose not to invest, we have the following:
(a) $x_{t}^{N}=2(\pi+b)$;
(b) $x_{t}^{N}=2(b-\pi)$;
(c) $x_{t}^{N}=0$. 
In cases (a) and (b) there is overinvestment relative the first-best. The NP's budget constraint is satisfied where $W(x)-B_{0}-\Pi_{0}=0$. Assuming that $W\left(x^{*}\right)>0$, if we raise $x$ above $x^{*}$ by a small enough amount, $W(x)$ will still be positive, but will fall in value. The solution given for cases (a) and (b) in (9) involves the NP raising $x$ so far above $x^{*}$ that $W(x)$ falls to zero. Thus, $x>x^{*}$. In case (c), however, $x_{t}^{N}$ equals the first-best level.

Our second proposition brings these results together and specifies whether, when there is underinvestment by and FP but overinvestment by an NP, welfare is higher in the former or the latter case.

Proposition 2 Consider traditional procurement for $b>\pi$ and $\bar{\Pi}=\Pi_{0}$. In cases (a) and (b) $x_{t}^{N}>x^{*}>x_{t}^{F}$, with welfare higher with an FP than with and NP. In case (c) $x_{t}^{N}=x^{*}=x_{t}^{F}$.

Proof. The rankings of $x_{t}^{F}, x_{t}^{N}$ and $x^{*}$ follow from (8) and (9). Now consider only (a) and (b). From (1), (2) and (3), $W^{\prime}(x)=\beta b+\gamma \pi-x$; and from (4), $W^{\prime}\left(x^{*}\right)=\beta b+\gamma \pi-x^{*}=0$. Since $W^{\prime \prime}(x)<0$, it follows that for any value of $x$ such that $W^{\prime}(x)<0$ we have that $x>x^{*}$, and for any value of $x$ such that $W^{\prime}(x)>0$ we have that $x<x^{*}$. Using Taylor expansions, given that $W^{\prime \prime \prime}(x)=0$, we have $W(x)=W\left(x^{*}\right)+W^{\prime}\left(x^{*}\right)\left(x-x^{*}\right)+W^{\prime \prime}\left(x^{*}\right)\left(x-x^{*}\right)^{2} / 2$. Since $W^{\prime}\left(x^{*}\right)=0$ and $W^{\prime \prime}(x)<0$, it follows that for $x=x_{1}$ and $x=x_{2}, W\left(x_{1}\right) \gtreqless W\left(x_{2}\right)$ as $\left(x_{1}-x^{*}\right)^{2} \lesseqgtr\left(x_{2}-x^{*}\right)^{2}$; that is, as $\left|x_{1}-x^{*}\right| \lesseqgtr\left|x_{2}-x^{*}\right|$. From (4), (8) and (9), in case (a) $\left|x_{t}^{F}-x^{*}\right|=|-(\pi+b) / 2|=(\pi+b) / 2$, while $\left|x_{t}^{N}-x^{*}\right|=\pi+b$. Hence, $\left|x_{t}^{F}-x^{*}\right|<\left|x_{t}^{N}-x^{*}\right|$, so that $W\left(x_{t}^{F}\right)>W\left(x_{t}^{N}\right)$. In case (b), $\left|x_{t}^{F}-x^{*}\right|=$ 
$|-(b-\pi) / 2|=(b-\pi) / 2$, while $\left|x_{t}^{N}-x^{*}\right|=|b-\pi|=b-\pi$. Hence, $\left|x_{t}^{F}-x^{*}\right|<$ $\left|x_{t}^{N}-x^{*}\right|$, so that $W\left(x_{t}^{F}\right)>W\left(x_{t}^{N}\right)$.

In welfare terms the FP weakly dominates the NP. With traditional procurement, social benefits are internalized to some extent by the FP because it bargains with the government, although there is underprovision compared to the first-best. In contrast, an NP prioritizes benefits and, as a result, overprovides relative to the first-best. We find that the NP overprovides to such an extent that welfare is lower than with an FP. We return to this result and its sensitivity to our assumptions in Section 3.

\subsection{PFI versus Traditional Procurement}

The above results can be used to give an overall comparison of the four institutional set-ups - with PFI or traditional procurement, and FP or NP provision. First, however, it is informative to note briefly whether with provision by a given type of firm (FP or NP) PFI or traditional procurement is preferable.

Lemma 3 Assume that $b>\pi$ and $\bar{\Pi}=\Pi_{0}$. (i) If provision is by an FP then traditional procurement is better than PFI in all cases. (ii) If provision is by an NP then in cases (a) PFI is better, while in cases (b) and (c) PFI and traditional procurement produce the same results.

Proof. These results follow immediately from the first-order conditions except for (ii) (a) and (b). Consider (ii)(a). Using the same approach as in the proof of Proposition 2 , since $x_{p}^{N}=2 \pi$ and $x_{t}^{N}=2(\pi+b)$, we have $\left|x_{p}^{N}-x^{*}\right|=|\pi-b|=b-$ $\pi$ and $\left|x_{t}^{N}-x^{*}\right|=\pi+b$. Hence, $\left|x_{p}^{N}-x^{*}\right|<\left|x_{t}^{N}-x^{*}\right|$, so that $W\left(x_{p}^{N}\right)>W\left(x_{t}^{N}\right)$. 
In (ii)(b), $x_{p}^{N}=0$ and $x_{t}^{N}=2(b-\pi)$, and so $\left|x_{p}^{N}-x^{*}\right|=|-(b-\pi)|=b-\pi$ and $\left|x_{t}^{N}-x^{*}\right|=b-\pi$. Hence, $\left|x_{p}^{N}-x^{*}\right|=\left|x_{t}^{N}-x^{*}\right|$, so that $W\left(x_{p}^{N}\right)=W\left(x_{t}^{N}\right)$.

It is clear from this lemma that the introduction of PFI has widened the scope for welfare-enhancing provision by and NP.

Our third proposition gives the arrangement, amongst the four options, that yields the greatest welfare.

Proposition 4 Assume that $b>\pi$ and $\bar{\Pi}=\Pi_{0}$. In case (a) welfare is highest with an NP and PFI if $b<3 \pi$, but with an FP and traditional procurement if $b>3 \pi$. In case (b) welfare is highest with an FP and traditional procurement. In case (c) welfare is lowest with an FP and PFI, but the other three arrangements all yield the first-best solution.

Proof. Case (a). From Propositions 1 and 2, either an NP with PFI, or an FP with traditional procurement, yields the highest welfare. Since $\left|x_{p}^{N}-x^{*}\right|=b-\pi$ and $\left|x_{t}^{F}-x^{*}\right|=(\pi+b) / 2$, we have that $\left|x_{p}^{N}-x^{*}\right| \gtreqless\left|x_{t}^{F}-x^{*}\right|$ as $b \gtreqless 3 \pi$. Thus, $W\left(x_{t}^{N}\right) \lesseqgtr W\left(x_{p}^{F}\right)$ as $b \gtreqless 3 \pi$. Cases (b) and (c) follow from Propositions 1 and 2 and Lemma 1.

\section{Alternative Assumptions}

In this section we examine the effects of dropping the assumptions that $b>\pi$ and $\bar{\Pi}=\Pi_{0}$. 


\section{$3.1 \quad b \leq \pi$}

Suppose that $b \leq \pi$; that is, assume that the dominant effect of an innovation is on profits, rather than benefits. (The assumption that $\bar{\Pi}=\Pi_{0}$ is retained.) Then a repeat of our earlier analysis gives the values of $x$ in shown Table 1 . Compared to our results for $b>\pi$, the values in the table only change for cases (b) and (c). However, the implications of the values in the table affect the welfare comparisons in all three cases. In case (b) each of the four arrangements now lead to the firstbest solution, so we focus on cases (a) and (c), highlighting the differences from our analysis for $b>\pi$.

\begin{tabular}{|l|l|l|l|l|l|}
\hline & $x^{*}$ & $x_{p}^{F}$ & $x_{p}^{N}$ & $x_{t}^{F}$ & $x_{t}^{N}$ \\
\hline (a) & $b+\pi$ & $\pi$ & $2 \pi$ & $(b+\pi) / 2$ & $2(b+\pi)$ \\
\hline (b) & 0 & 0 & 0 & 0 & 0 \\
\hline (c) & $\pi-b$ & $\pi$ & 0 & $(\pi-b) / 2$ & 0 \\
\hline
\end{tabular}

Table 1. Levels of $x$ when $b>\pi$ and $\bar{\Pi}=\Pi_{0}$

Consider PFI first. In case (a), NP provision now leads to overinvestment. As a result, welfare under PFI is now found to be higher with an FP than with an NP. ${ }^{4}$ In case (c) the levels of investment by an FP and an NP are each the same as when $b>\pi$, but the first-best investment $x^{*}$ has changed. It is found that $W\left(x_{p}^{F}\right) \gtreqless W\left(x_{p}^{N}\right)$ as $\pi \gtreqless 2 b .^{5}$

Turning to traditional procurement, the analysis of case (a) is identical to that in the previous section: $x_{t}^{N}>x^{*}>x_{t}^{F}$, with welfare higher with an FP than an NP. In case (c) $x^{*}>x_{t}^{F}>x_{t}^{N}$ and so welfare is also higher with an FP than an NP.

${ }^{4}\left|x_{p}^{F}-x^{*}\right|=b$ and $\left|x_{p}^{N}-x^{*}\right|=(b+\pi) / 2$. Since $b \leq \pi$, we have that $\left|x_{p}^{F}-x^{*}\right| \leq\left|x_{p}^{N}-x^{*}\right|$. Therefore $W\left(x_{p}^{F}\right) \geq W\left(x_{p}^{N}\right)$.

${ }^{5}\left|x_{p}^{F}-x^{*}\right|=b$ and $\left|x_{p}^{N}-x^{*}\right|=\pi-b$. The welfare ranking in the text follows. 
If provision is to by FP then in case (a), for both PFI and traditional procurement, there will be underprovision relative to the first-best, but we now find that PFI is preferred to traditional procurement. In case (c) it is found that $W\left(x_{p}^{F}\right) \gtreqless W\left(x_{t}^{F}\right)$ as $\pi \gtreqless 3 b ;^{6}$ that is, if the profit-effect of investment substantially outweighs the benefit-effect, PFI with an FP is preferred to traditional procurement with an FP. Alternatively, if provision is to be by an NP then in case (a) there is overinvestment, particularly under traditional procurement, so PFI is preferred. In case (c), however, neither PFI nor traditional procurement results in any investment.

The overall implications for the choice between the four arrangements are summarized in the next proposition.

Proposition 5 Assume that $b \leq \pi$ and $\bar{\Pi}=\Pi_{0}$. In case (a) welfare is highest with an FP under PFI. In case (b) all arrangements yield the first-best. In case (c), if $\pi>3 b$ welfare is highest with an FP under PFI, while if $\pi<3 b$ welfare is highest with an FP under traditional procurement.

A significant feature to emerge from Propositions 3 and 4 is that, while PFI has opened up new opportunities for welfare-enhancing public service provision NPs, NP provision can only be strictly preferred if $b>\pi$.

\section{$3.2 \quad \bar{\Pi} \neq \Pi_{0}$}

We have assumed until now that the research into innovation and the subsequent implementation cannot be a net cost to the NP; that is, the amount of profit ${ }^{6}\left|x_{p}^{F}-x^{*}\right|=b$ and $\left|x_{t}^{F}-x^{*}\right|=(\pi-b) / 2$. Therefore $\left|x_{p}^{F}-x^{*}\right|-\left|x_{t}^{F}-x^{*}\right|=(3 b-\pi) / 2$, and
the condition in the text follows. 
from fulfilling the basic standards must be at least achieved by innovation and implementation. Suppose, however, that the NP may be willing to forgo all of $\Pi_{0}$ to increase benefits, where, by assumption, $\Pi_{0} \geq 0$. Thus, we have $\bar{\Pi}=0$ in (??). ${ }^{7}$ For brevity, we refer to this case as entailing a 'less tight' budget constraint - compared to the case analyzed in Section 3.

Assume first that $b>\pi$ and consider PFI. In case (a) the NP now invests up to the point at which $\pi x_{p}^{N}-\left(x_{p}^{N}\right)^{2} / 2=-\Pi_{0}$. Thus, taking the real root of the quadratic,

$$
\text { (a) } x_{p}^{N}=\pi+\left(\pi^{2}+2 \Pi_{0}\right)^{1 / 2} \text {. }
$$

Hence, having the less tight budget constraint causes the NP to invest more under PFI. In case (b), $x$ increases benefits, and although it is unprofitable, the availability of the amount $\Pi_{0}$ to spend enables the NP to invest. In this case $-\pi x_{p}^{N}-\left(x_{p}^{N}\right)^{2} / 2=-\Pi_{0}$. Again taking the real root,

$$
\text { (b) } x_{p}^{N}=-\pi+\left(\pi^{2}+2 \Pi_{0}\right)^{1 / 2} \text {. }
$$

As in case (a), in case (b) the less tight budget constraint results in greater investment. In case (c), however, since investment reduces benefit, the NP sets $x_{p}^{N}=0$.

With traditional procurement, in cases (a) and (b) the NP's budget constraint binds with $z=b x$; but now this entails $b x+\gamma \pi x-x^{2} / 2=-\Pi_{0}$. Thus we obtain

$$
x=b+\gamma \pi+\left[(b+\gamma \pi)^{2}+2 \Pi_{0}\right]^{1 / 2} .
$$

\footnotetext{
${ }^{7}$ Other levels of constraint might also occur. For example, the NP might have other urgent calls on its funds, so that, net, it must accumulate some profit. Alternatively, it may be willing to forgo some, but not all, of $\Pi_{0}$. We focus on the specific constraint in the text for simplicity, but the effects of other levsl of the constraint may be inferred from our results.
} 
We therefore have

$$
\begin{aligned}
& \text { (a) } x_{t}^{N}=b+\pi+\left[(b+\pi)^{2}+2 \Pi_{0}\right]^{1 / 2}>2(b+\pi) \\
& \text { (b) } x_{t}^{N}=b-\pi+\left[(b-\pi)^{2}+2 \Pi_{0}\right]^{1 / 2}>2(b-\pi) .
\end{aligned}
$$

In case (c) the NP will choose not to invest.

These results give our next lemma.

Lemma 6 Assume that $b>\pi$ and that the NP aims to break even overall (a 'less tight budget constraint'). (i) In cases (a) and (b) under both PFI and traditional procurement the NP will invest more with the less tight budget constraint. (ii) In case (a), for $b<3 \pi$, if $\Pi_{0}$ is not too large, then having a less tight budget constraint this increases the extent to which the NP under PFI yields greater welfare than other arrangements do; but, for increases in $\Pi_{0}$ above $\left(b^{2}-\pi^{2}\right) / 2, d W\left(x_{p}^{N}\right) / d \Pi_{0}<$ 0 , and if $\Pi_{0}$ becomes large enough, $W\left(x_{f}^{F}\right)>W\left(x_{p}^{N}\right)$. If $b>3 \pi, W\left(x_{f}^{F}\right)>W\left(x_{p}^{N}\right)$ for all $\Pi_{0} \geq 0$. (iii) In cases (b) and (c), having a less tight budget constraint has no implications for which arrangement yields the highest welfare.

Proof. (i) This follows from comparison of (10) with (6), and (11) with (7). (ii) $b<3 \pi$ is the condition for which $x_{p}^{N}$ is the best arrangement in Proposition 3. From (4) and (10), $x_{p}^{N}=x^{*}$ if $\Pi_{0}=\left(b^{2}-\pi^{2}\right) / 2$. A larger $\Pi_{0}$ than this raises $x_{p}^{N}$ above $x^{*}$, and eventually $W\left(x_{p}^{N}\right)$ becomes smaller than $W\left(x_{f}^{F}\right)$. If $b>3 \pi$, we already have that $W\left(x_{f}^{F}\right)>W\left(x_{p}^{N}\right)$ for $\Pi_{0}=0$; a higher value of $\Pi_{0}$ strengthens this inequality. (iii) In case (b), if $\Pi_{0}=0, x_{p}^{N}>x^{*}$ and an NP under traditional procurement is not the preferred arrangement. Since $d x_{p}^{N} / d \Pi_{0}>0, d W\left(x_{p}^{N}\right) / d \Pi_{0}<0$, so that 
this arrangement is still not preferred. In case (c) since $x_{p}^{N}=0$ for all $\Pi_{0} \geq 0$, $d w\left(x_{p}^{N}\right) / d \Pi_{0}=0$

Since $d x_{p}^{N} / d \Pi_{0}>0$, the existence of a less tight budget constraint can have a positive effect on welfare if $x_{p}^{N}<x^{*}$ when (as in Section 3) the budget constraint is tight. However, a sufficiently large value of $\Pi_{0}$ can have a negative effect on welfare by causing excessive investment by the NP under PFI.

If, instead, $b \leq \pi$, no changes are required to our conclusions in the previous sub-section about which form of provision yields the greatest welfare.

\section{Illustrations}

In the light of these results, we now discuss some examples, applying our results to highlight circumstances where one institutional arrangement is preferable to another.

\section{Case 7 Profitable quality improvement}

Investment in building quality can raise both social benefit and reduce maintenance costs. For example, better school buildings with less frequent need for repairs also lead to fewer disruptions and help to create a good learning environment; and higher-quality hospital buildings reduce disruptions and generate a better healing environment. Construction of roads is another example where investment can raise both profit and benefit. In all these cases, our results suggest that the use of PFI is desirable. If the effect of investment on maintenance cost is relatively large, welfare will be maximized maximized by FP provision; but if the effect of investment on maintenance is relatively small, NP provision is preferable. 
It is interesting to note that it is precisely for building schools, roads and hospitals that PFI with an FP is being used in the UK. By bundling the building and maintenance functions of infrastructure provision, PFI gives incentives to the contractor to internalize the effect of its investment on maintenance cost. As contracts tend to be long-lived (generally about 25 years), better design has the potential to translate into significantly greater profits, by reducing the future stream of maintenance cost.

The profitable quality improvement scenario may also apply for free-standing projects, such as leisure centres and nursing homes, where users are charged a fee and where there is competition among providers, so that a higher quality of service may well raise total revenues and profits. Thus, also for these types of services, provision by PFI is desirable and if a rise in quality is the dominant effect of investment, the use of an NP is preferred.

\section{Case 8 Unprofitable quality improvement}

Investment in building quality that raises social benefit can also result in lower profit because a better design may be expensive to implement and maintain. Furthermore, many public services are characterized by an inelastic demand and are offered in conditions of limited competition among the private providers. If also the government is the purchaser of the service or if user fees are specified in advance, increasing some unverifiable quality aspect of the service is likely to be unprofitable for the contractor.

In these circumstances our analysis indicates that the weakly welfare-maximizing arrangement is traditional procurement with an FP. In this context, it is interesting 
to note that the NHS Confederation in the UK recently reported that PFI hospitals designed and built by FPs often failed to create a good healing environment with less noise and more daylight. ${ }^{8}$

Case 9 Cost cutting at the expense of quality

Investments that decrease costs may have the side-effect of reducing social benefit. This may be in the form of reduced safety, for example in railway maintenance or air traffic control, but may relate to any quality aspect of the service (e.g. quality of health care). In this case our analysis indicates that the preferred arrangement for provision is highly senstive to parameter values. If, however, the first-best solution is to have no such investments, then provision by an NP, either through PFI or traditional procurement, is the weakly preferred arrangement.

In the UK the healthcare system is changing fast, and significant parts of provision are being put in private hands. If there is concern that provision by FPs will lead to lower welfare through cost cutting at the expense of quality, this concern may be alleviated by reliance on NPs. If we broaden our analysis to allow for the possibility that firms may have more than one option as to whcih kind of investment they make, investment in quality improvement also being feasible, then the broad indication of our analysis is that, with provision by an NP, PFI may be preferable to traditional procurement.

\footnotetext{
${ }^{8}$ See PublicPrivateFinance, 85, July/August 2004.
} 


\section{Conclusions}

In this paper we have analyzed contracting out to a not-for-profit firm and to a for-profit firm under two alternative procurement arrangements. The first PFI, whereby the firm is allocated control rights over how to deliver the service; the second is traditional procurement, whereby the government retains control rights.

PFI increases the scope for not-for profit provision, compared to traditional procurement; that is, in some scenarios, the optimal administrative arrangement is PFI with a not-for-profit firm, even though, if traditional procurement were used, it would be preferable to use a for-profit firm.

\section{References}

[1] Bennett, J., Iossa, E., Legrenzi, G., 2003. The role of commercial non-profit organizations in the provision of public services. Oxford Review of Economic Policy, 19, 335-347.

[2] Bennett, J., Iossa, E., 2006. Building and managing facilities for public services. Journal of Public Economics, 90, 2143-2160.

[3] Benz, A., Grout, P. A., Halonen, M., 2001. What should the state buy? CMPO Working Paper No. 01/40, University of Bristol.

[4] Besley, T., Ghatak, M., 2001. Government versus private ownership of public goods. Quarterly Journal of Economics, 116, 1343-1372. 
[5] Bös, D., De Fraja, G., 2002. Quality and outside capacity in the provision of health services. Journal of Public Economics, 84, 199-218.

[6] Glaeser, E. L., Shleifer, A., 2001. Not-for-profit entrepreneurs. Journal of Public Economics, 81, 99-115.

[7] Hansmann, H., 1986. The role of the nonprofit enterprise. Yale Law Journal, 89, 835-901.

[8] Hansmann, H., 1996. The Ownership of Enterprise. Harvard University Press, Cambridge, MA and London, UK.

[9] Hart, O., 1995. Firms, Contracts and Financial Structure. Clarendon Press, Oxford, UK.

[10] Hart, O., 2002. Incomplete contracts and public ownership: remarks and an application to public-private partnerships,' mimeo, Harvard University.

[11] Hart, O., Shleifer, A., Vishny, R. W., 1997. The proper scope of government: theory and an application to prisons. Quarterly Journal of Economics, 112, 1119-1158.

[12] HM Treasury, 2003. PFI: Meeting the Investment Challenge. The Stationery Office, London, UK.

[13] IPPR, Institute of Public Policy Research, 2003. In the Public Interest? Assessing the potential for Public Interest Companies. 
[14] King, S., Pitchford, R., 2001. Private or Public? A Taxonomy of Optimal Ownership and Management Regimes. Mimeo.

[15] Rose-Ackerman, S., 1996. Altruism, nonprofits, and economic theory. Journal of Economic Literature, 34, 701-728.

[16] Schmitz, P. W., 2000. Partial privatization and incomplete contracts: the proper scope of government reconsidered. FinanzArchiv, 57, 394-411.

[17] Weisbrod, B., 1997. The future of the nonprofit sector: its entwining with private enterprise and government. Journal of Policy Analysis and Management, $16,541-555$. 